\title{
AN APPLICATION OF $K$-THEORY TO EQUIVARIANT MAPS
}

BY JAMES W. VICK

Communicated by P. Emery Thomas, April 18, 1969

1. Introduction. Let $p$ be a prime and $Z_{p}$ the cyclic group of order $p$. Denote by $\left(S^{2 n+1}, T_{p}\right)$ the free action of $Z_{p}$ on $S^{2 n+1}$ given by

$$
T_{p}\left(z_{1}, \cdots, z_{n+1}\right)=\left(\lambda z_{1}, \cdots, \lambda z_{n+1}\right) \quad \text { where } \lambda=\exp (2 \pi i / p) .
$$

Then given any pair $(X, T)$ consisting of a finite complex $X$ and a fixed point free transformation $T: X \rightarrow X$ of period $p$, one might ask for the least value of $n$ for which there is an equivariant map of $(X, T)$ into $\left(S^{2 n+1}, T_{p}\right)$. Questions of this type have been previously investigated [3], [4], [5] with particular emphasis on the case $p=2$.

It is the purpose of this note to describe a method for using $K$ theory to approach this problem for certain actions on lens spaces.

2. Preliminaries. Let $B Z_{p^{r}}$ be a classifying space for the group $Z_{p^{r}}$, taken to be a CW complex whose odd dimensional skeleta are the lens spaces $L\left(p^{r}, 2 n+1\right)=S^{2 n+1} / T_{p^{r}}$ where $T_{p^{r}}$ is defined analogously to $T_{p}$. Let $B S^{1}$ be a classifying space for the circle group whose even dimensional skeleta are complex projective spaces $C P(n)$.

Denote by $K^{*}$ and $K_{*}$ the $Z_{2}$-graded cohomology and homology theories arising from the unitary spectrum (see [6] for details).

(2.1) TheOREm. There is a short exact sequence of groups

$$
0 \rightarrow K^{0}\left(B S^{1}\right) \stackrel{\alpha}{\rightarrow} K^{0}\left(B S^{1}\right) \stackrel{\gamma}{\rightarrow} K^{0}\left(B Z_{p^{r}}\right) \rightarrow 0
$$

where $K^{0}\left(B S^{1}\right) \approx Z[[\mu]]$ is a power series ring in one variable, $\gamma$ is a ring homomorphism, and $\alpha$ is given by multiplication by $\left[(\mu+1)^{p^{r}}-1\right]$.

(2.2) THEOREM. There exists a short exact sequence of groups

$$
0 \rightarrow \tilde{K}_{0}\left(B S^{1}\right) \stackrel{\beta}{\rightarrow} K_{0}\left(B S^{1}\right) \rightarrow K_{1}\left(B Z_{p^{r}}\right) \rightarrow 0
$$

where $K_{0}\left(B S^{1}\right)$ is the free abelian group generated by $\left\{g_{i}\right\}_{i=0}^{\infty}$ and

$$
\beta\left(g_{k}\right)=g_{k-p^{r}}+\left(\begin{array}{c}
p^{r} \\
1
\end{array}\right) g_{k-p^{r}+1}+\cdots+\left(\begin{array}{c}
p^{r} \\
p^{r}-1
\end{array}\right) g_{k-1},
$$

where $g_{k}=0$ whenever $k<0$. 
(2.3) CoRollary. $K^{0}\left(B Z_{p^{r}}\right) \approx Z[[\mu]] / I$ where $I$ is the principal ideal generated by $\left[(\mu+1)^{p^{r}}-1\right]=\psi^{p^{r}}(\mu)$, where $\psi^{p^{r}}$ is the Adams operation [1].

These groups have been previously computed [2] in terms of completions of representation rings.

The sequence in (2.2) allows us to express $K_{1}\left(B Z_{p^{r}}\right)$ in terms of generators and relations. This is convenient because there is a close relationship between the generators and the skeletal decomposition of $B Z_{p^{r}}$. Specifically, $g_{k}$ is in the image of $i_{*}$, where $i$ is the inclusion of the $m$-skeleton in $B Z_{p^{r}}$, if and only if $m \geqq 2 k+1$.

Denote by $Z(1 / p)$ the ring of all rational numbers with denominator a power of $p$. Let $Z(1 / p ; \lambda, r)$ be the ring of polynomials in $\lambda$ with coefficients in $Z(1 / p)$ subject to the relation $1+\lambda+\lambda^{2}+\cdots$ $+\lambda^{p^{r}-1}=0$. Finally, let $Z(\lambda, r)$ be the subring of $Z(1 / p ; \lambda, r)$ consisting of those polynomials with integer coefficients.

(2.4) Corollary. There is an isomorphism of groups

$$
K_{1}\left(B\left\llcorner_{p}\right) \approx Z(1 / p ; \lambda, r) / Z(\lambda, r) .\right.
$$

From examining these structures, we have the following observation:

(2.5) Corollary. $\tilde{K}^{0}\left(B Z_{p}\right)$ is a compact group, and is in fact isomorphic to the character group of the discrete group $K_{1}\left(B Z_{p}\right)$.

This appears to be true as well for the prime power case, although the verification is considerably more complex. Aspects of this duality are the subject of current study.

3. Application. Let $\phi: Z_{p^{r} \rightarrow Z_{p}}$ be the homomorphism given by reducing $\bmod p$. Then there is associated to $\phi$ a homotopy class of maps $f: B Z_{p} \rightarrow B Z_{p}$, and we take $f$ to be a representative of that homotopy class. Let

$$
f_{*}: K_{1}\left(B Z_{p^{r}}\right) \rightarrow K_{1}\left(B Z_{p}\right)
$$

be the map induced by $f$. Then denoting the generators of $K_{1}\left(B Z_{p}{ }^{r}\right)$ by $\left\{h_{i}\right\}_{i=0}^{\infty}$ and the generators of $K_{1}\left(B Z_{p}\right)$ by $\left\{g_{i}\right\}_{i=0}^{\infty}$, there is the following fact about $f_{*}$.

(3.1) Proposition. (a) $f_{*}\left(h_{j}\right)=g_{k}+$ terms of lower index if $j=k \cdot p^{r-1}$,

(b) $f_{*}\left(h_{j}\right)=$ terms of index lower than $j / p^{r-1}$ if $p^{r-1}$ does not divide $j$.

(3.2) CoROLlary. No representative of the homotopy class of $f$ can map the $2 j+1$ skeleton of $B Z_{p^{r}}$ into the $2 k-$ skeleton of $B Z_{p}$ if $j=k \cdot p^{r-1}$. 
Now observe that since $\left(T_{p^{r+1}}\right)^{p}=T_{p^{r}}$, the transformation

$$
T_{p^{r+1}}: S^{2 n+1} \rightarrow S^{2 n+1}
$$

induces a fixed point free transformation

$$
T: S^{2 n+1} / T_{p^{r}} \rightarrow S^{2 n+1} / T_{p^{r}} \text { of period } p .
$$

This gives a pair $\left(L\left(p^{r}, 2 n+1\right), T\right)$ in the previous sense. As an immediate consequence of (3.2) we have the following

(3.3) Corollary. Let $n=k \cdot p^{r}$ and suppose there exists an equivariant map

$$
\left(L\left(p^{r}, 2 n+1\right), T\right) \rightarrow\left(S^{2 m+1}, T_{p}\right),
$$

then $m \geqq k$.

The motivation for seeking new tools in approaching this problem is the failure of the standard cohomological invariants to yield much useful information. The cohomology of these lens spaces is generated by one and two dimensional elements; hence the information given by the reduced $p$ th powers comes completely from the ring structure. If $\bar{f}: L\left(p^{r+1}, 2 n+1\right) \rightarrow L(p, 2 m+1), r \geqq 1$, is the map induced on the quotient spaces from some equivariant map and $\bar{f}^{*}$ is the associated homomorphism in cohomology, then the square of the image of a generator under $\bar{f}^{*}$ must be zero.

Much appreciation is due Professor E. E. Floyd for his advice in the preparation of this work.

\section{REFERENCES}

1. J. F. Adams, Vector fields on spheres, Ann. of Math (2) 75 (1962), 603-632.

2. M. F. Atiyah, Characters and cohomology of finite groups, Inst. Hautes Etudes Sci. Publ. Math. 9 (1961), 23-64.

3. P. E. Conner and E. E. Floyd, Fixed point free involutions and equivariant maps, Bull. Amer. Math. Soc. 66 (1960), 416-441.

4. - Fixed point free involutions and equivariant maps. II, Trans. Amer. Math. Soc. 105 (1962), 222-228.

5. - Periodic maps which preserve a complex structure, Bull. Amer. Math. Soc. 70 (1964), 574-579.

6. A. Hattori, Integral characteristic numbers for weakly almost complex manifolds, Topology 5 (1966), 259-280.

Princeton University, Princeton, New Jersey 08540 